\title{
Secondary Mandibular Deformity Correction in TMJ Ankylosis Patients Using Distraction Osteogenesis
}

\author{
Dr. S Gandhiraj ${ }^{1}$, Dr.Akash Kasatwar ${ }^{2}$, Dr. Sandeep Agarwal ${ }^{3}$, \\ Dr. K Subalakshmi ${ }^{4}$, Dr. Sandeep Patil ${ }^{5}$, Dr. Jayshri Nandanwar ${ }^{6}$ \\ ${ }^{1}$ (Former Professor \& HOD, Dept of Oral \& Maxillofacial Surgery, Sathyabama University Dental College \& \\ Hospital, Chennai 600119), \\ ${ }^{2}$ (Assistant Professor \& Dept of Oral \& Maxillofacial Surgery, Sharad Pawardental college \& hospital, \\ DMIMS, Wardha, Maharashtra, India), \\ ${ }_{3}^{3}$ Consultant Oral \& Maxillofacial Surgeon, Mumbai), \\ ${ }_{4}^{4}$ (Senior Assistant Professor, Dept of dental surgery Government Tiruvannamalai medical college and hospital , \\ Tiruvannaimalai,Tamilnadu,India ), \\ ${ }^{5}$ (Assistant Professor, Department of Oral and Maxillofacial Surgery, B. V.D.U. Dental College and Hospital, \\ Sangli. 416414, Maharashtra, India), \\ ${ }^{6}$ (Assistant Professor, Dept of Periodontology \&Implantology, RRK Dental College \\ \&Hospital,Akola,Maharashtra,India),
}

\begin{abstract}
TMJ Ankylosis is a devastating condition occurs secondary to trauma. Patient with Tmjankylosis not only have difficulty in mouth opening but also have marked facial deformity. Various treatment modalities are available for the management of this deformity like release of ankylotic mass reconstruction; primary distraction Osteogenesis and secondarily release of ankylotic mass.The aim of this study was to evaluate the correction of facial deformity using distraction osteogenesis principlesIn the present study total of five patients were selected. In all the patients, in first stage TMJ ankylotic mass were removed. In a second stage distraction Osteogenesis were performed on the affected side for the correction of facial deformity. Distraction Osteogenesis after release of TMJ ankylosis is a feasible and reliable technique for the correction of secondary mandibular deformity.
\end{abstract}

Key words: tmjankylosis, facial asymmetry, distraction osteogenesis

\section{Introduction}

Temporo-mandibular joint ankylosis is a common cause of acquired mandibular deformity in children and adults. [1] The most frequent causes of condylar damage are trauma to the joint and infections ${ }^{2}$. The damage to the condyle in early childhood period may result in facial asymmetry due to alteration in mandibular growth.In general, younger the patient at the time of condylar damage and longer the period of ankylosis before treatment, the more severe will be the degree of deformity. The deformity is the result of both the condylar destruction and the lack of stimulus to the growth induced by mandibular function and movements. There are various techniques available to release the ankylosis but the correction of the resulting facial deformity in these cases is still a challenge . Treatment techniques range from two stage management that is ankylosis release followed by correction of secondary deformities in the second stage, to one stage correction of mandibular skeletal deformities along with ankylosis .[3,4]

Numerous surgical procedures have been advocated to correct facial deformities include, costochondral grafts, mandibular osteotomies with or without bone grafts. [2, 4,5] Some of these procedures may be followed by relapses, over growth as seen with costochondral graftswith added donor site morbidity.[2] Also sometimes these procedures do requires several operations to achieve desired correction. The overlying soft tissues also pose a problem to adapt adequately in these modalities contributing to increase chances of relapses.More recently, distraction Osteogenesis(DO) has been proposed for the correction of secondary mandibular deformities due to ankylosis of the temporomandibular joint.[6]Distraction osteogenesis of the human mandible was introduced in 1992 by McCarthy et al \& subsequently several reports on successful mandibular lengthening have been published[7].Surgeons are now able to generate new bone in patients with a bilateral or unilateral mandibular body deficiency or severely hypoplastic ascending ramus when it is missing in ankylotic patient. It probably represents the first tissue engineering surgical techniques applied in the craniofacial field. It is gaining more momentum in the treatment of deformity correction because of its unique advantages. The aim of this study is to evaluate the efficacy of distraction technique in correcting secondary mandibular deformity in temporo mandibular joint ankylosis patients by using an indigenously designed external distraction device. 


\section{Materials And Methods}

This clinical study was done in the department of oral \& maxillofacial surgery, Tamil Nadu Government Dental College \& Hospital, Chennai. The criteria for selection of patients include Mandibular uni/bilateral body or ramus hypoplasia secondary to TMJ ankylosis in adult patients, who were treated for TMJ ankylosis with temporalis myofascial flap interpositioning arthroplasty as a separate first stage operation 3-4 months prior to distraction. A group of four patients were selected, three male and one female with age ranging from (19-28 year). All were having unilateral TMJ ankylosis, for which temporalis myofascial flap interpositional arthroplasty was done in all the cases. All the four patients underwent unilateral distraction with extraoral uniplanar distraction device placed with an oblique vector for simultaneous lengthening of both ramus and body.I Evaluation of the patient included a thorough and detailed history, clinical examination, facial photographs radiographic examination, frontal cephalogram (Grummon'sanalysis) and lateral cephalogram (Bork's analysis)[Figure 1]. Measurement of vertical height of ramus and horizontal length of the mandible done both on the affected and unaffected side, and the deficiency on the affected side was recorded using OPG, 3D CT scan, lateral and frontal cephalograms. Assessment of the osteotomy cut and vector planning was done using OPG as a guide. For vertical (ramus) as well as horizontal (body) lengthening, osteotomy cut was planned approx. $1 \mathrm{~cm}$ superior to gonion extending up to external oblique ridge in the retromolar region just distal to last molar. Oblique vector for device placement was planned over pre- determined osteotomy cut; this vector is oblique in relation to occlusal plane and parallel in relation to ascending ramus. Depending on the Deformity and other anatomical restrictions modifications were made, if necessary.inferior alveolar canal distance from the lower border of mandible was marked on tracing paper using opg as a guide. This is done in order to avoid injury to inferior alveolar nerve during pins placement.

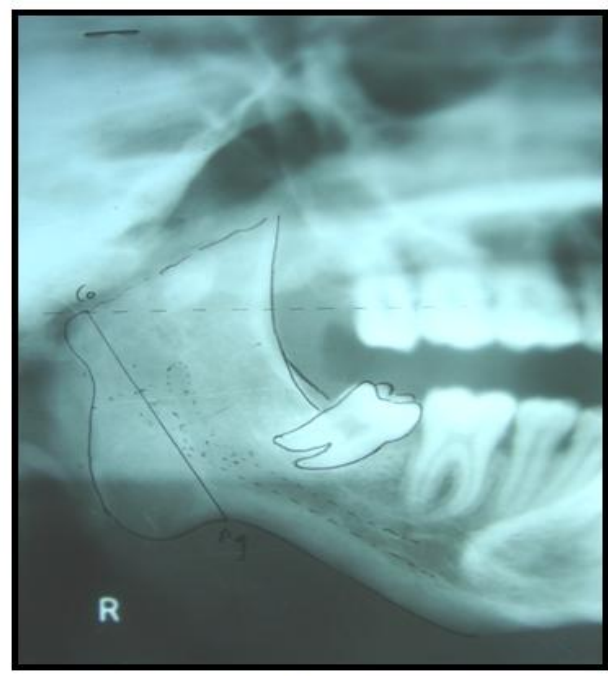

A

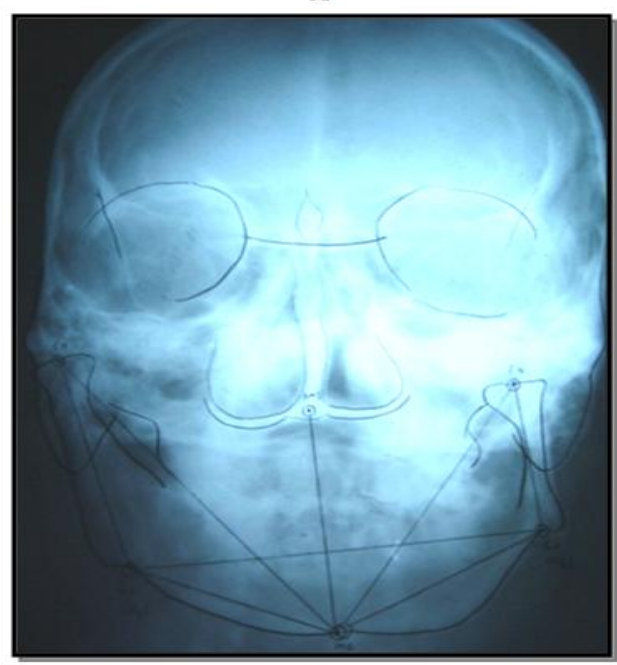

C

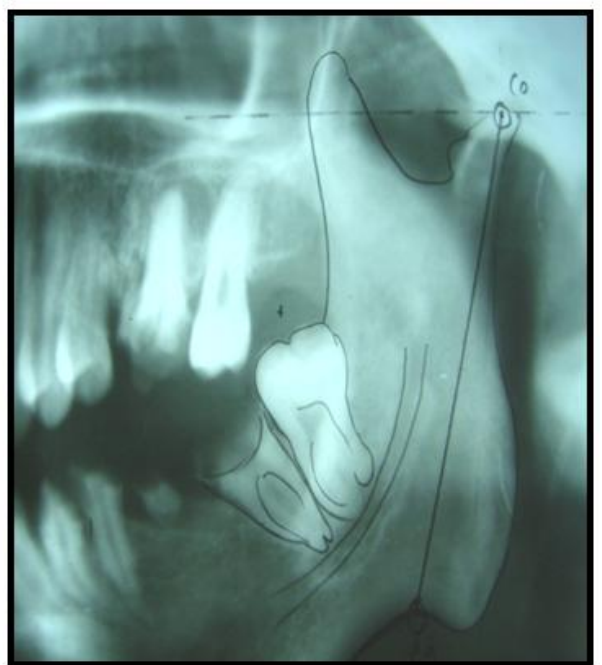

B

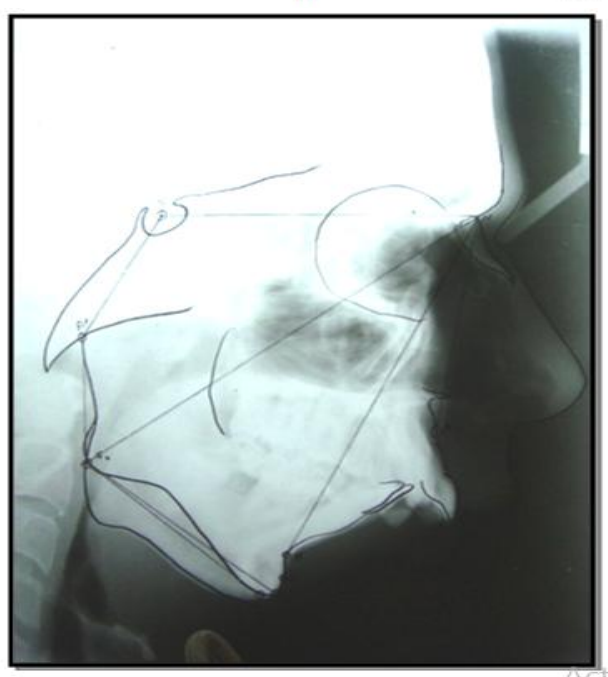

$\mathrm{D}$

Fig 1, A. Affected Rt Side Of The Mandible, B.Unaffected Lt Side Of The Mandible, C.Grummon Analysis, D.Bjork Analysis 


\section{Surgical Technique}

Naso endotracheal intubation was used for all patients. It was inserted either by blind nasal or fibreoptic technique depending upon the difficulty during intubation. Skin incision was given $2 \mathrm{~cm}$ below angle and lower border of mandible approx $3-4 \mathrm{~cm}$ in length parallel to skin creases. Layer wise dissection done exposing deformed angle and body region. Using bone markers, osteotomy cut marked after calculating the distance using calliper both on the superior and inferior region as planned presurgically. Following this another marking was made indicating vector for device placement

[Figure 2].Corticotomy was done using saw on the buccal cortex. Then through the buccal cortical cut it is continued to the superior $1 / 3^{\text {rd }}$ and the inferior $1 / 3^{\text {rd }}$ of the lingual cortex, maintaining a part of cortical bone over inferior alveolar region lingually. After completion of the corticotomy acrylic template was placed over the planned vector line and four pin holes were made using 701 bur. Now once the corticotomy is completed, intramedullary bicortical pin placement is done using Pin key. Care has been taken to insert the pin through skin using small stab incision by 11 no. blade so that scar marks are left in more lower inconspicuous area, leaving sufficient area for suturing tissues without tension. Once the pins are placed in position, the distraction device is positioned and fixed temporarily. Now using a thin osteotome, the lingual cortex was fractured gently by taking immense care to protect the integrity of the neurovascular bundle. Slight activation of the distraction device was done in order to confirm the discontinuity between distal and proximal segments after osteotomy. After confirming the successful separation of the segments device is kept in previous inactivated position.Layerwise closure of the surgical wound is then done using 3-0 vicryl for pterygomasseteric sling, fascia and subcutaneous tissues and 3-0 prolene / silk for skin. After a latency period of 5 to 7 days, distraction was done at the rate $1 \mathrm{~mm} /$ day with a rhythm of $0.5 \mathrm{~mm}$ two times a day was maintained. The activation was done till the desired length needed for correction and maximum midline shift of affected side deviated chin was achieved. The device was left in situ for a consolidation period of 6-8 weeks till the evidence of bone formation is seen on the radiographs. After the consolidation phase the device was removed under local anaesthesia. All the patients were asked to report to the outpatient department for radiographic assessment once in a month to see the amount of bone formation. The follow up period ranges from minimum of 3 month to maximum of 6 months.

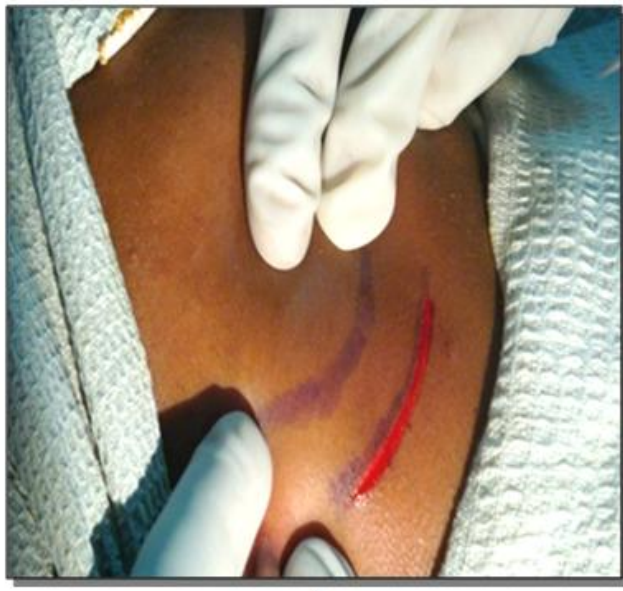

A

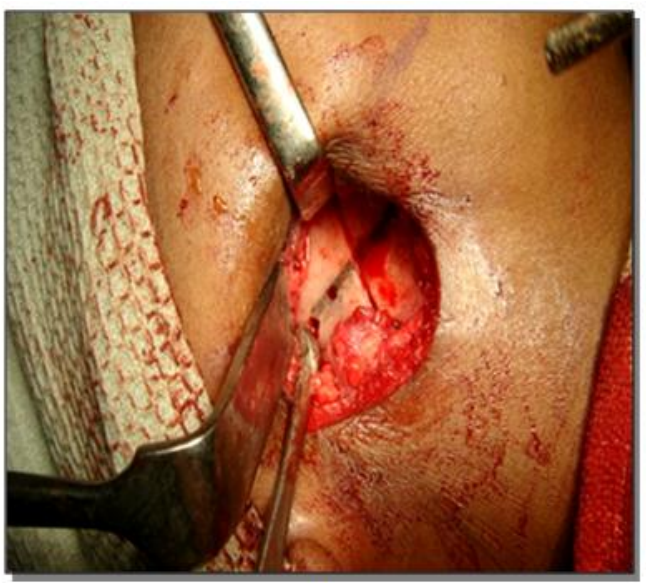

C

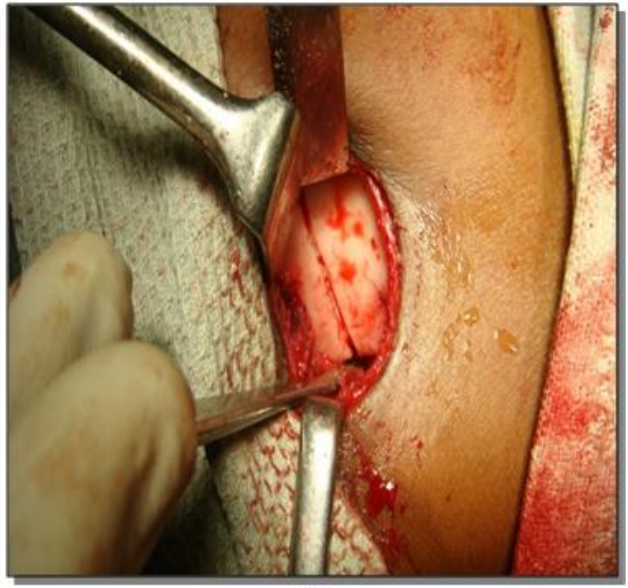

B

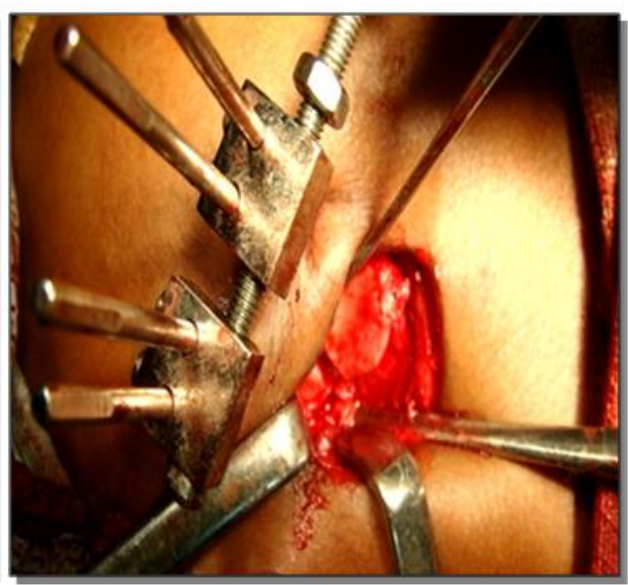

$\mathrm{D}$ 

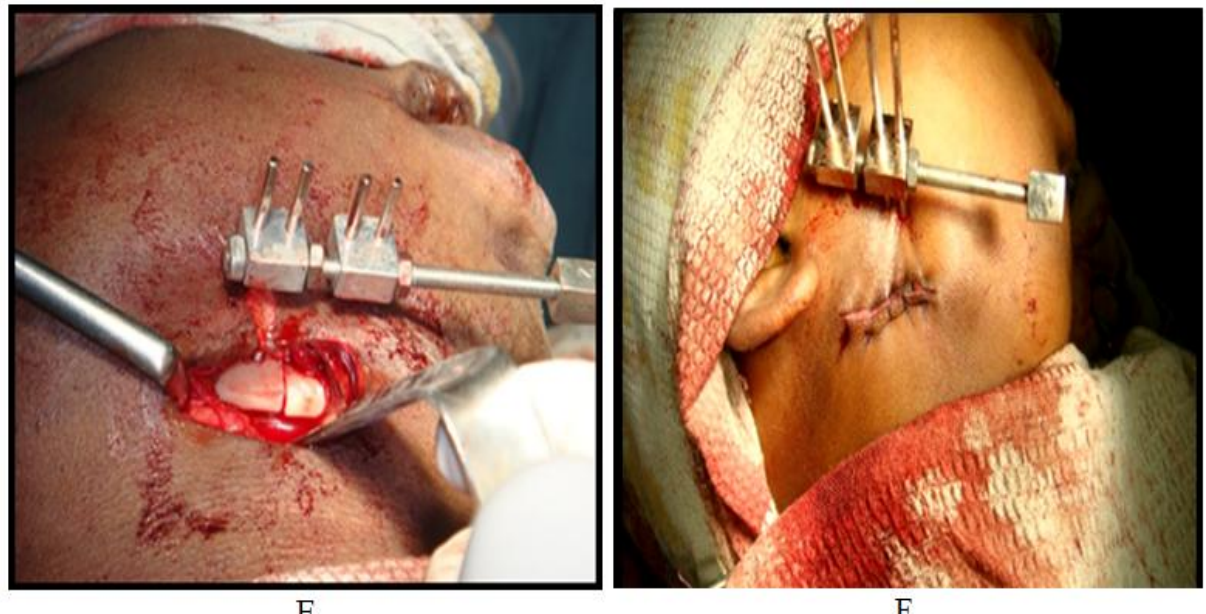

Fig 2, A, Submandibular Incision \& Exposure of the Angle Region After Layerwise Dissection, B.Osteotomy Cut At Angle Region,C.Pinhole Placed Over The Planned Vector Line Using Acrylic Template,D.Completition Of Osteotomy Cut With Thin Osteotome\& Placement Of Distraction Device,E. Both Distal And Proximal Segment Kept In Inactivated Position, F.Skin Closure

\section{Observation And Results}

The use of an indigenously and custom designed extra oral distraction device for the correction of mandibular deformities showed good results[Table 1]. Of the four patients included in this study, we performed oblique distraction for both ramus and body lengthening unilaterally. Approximately 18 to $25 \mathrm{~mm}$ of lengethening were needed in all the patients. Lengthening achieved in all the patients with some minor discrepancy. The discrepancy observed in one case, where device was placed obliquely was due to bending of device at the distraction-compression unit during activation, where as in other patient with oblique vector we achieve desired vertical ramus lengthening but not horizontal body lengthening to the same extent. At the end of desired lengthening, all the patients except one showed good facial aesthetics. There was a good chin projection with movement of the chin towards the unaffected side coinciding with facial midline. We also observed better soft tissue profile with expansion post distraction in all patients. Radiographs were taken periodically to assess these patients. Radiographs showed evidence of bone formation taken at the end of consolidation period. Temporo mandibular joint disturbances, Inferior alveolar nerve complication like paraesthesia were not encountered in our study. All the patients are followed periodically for evaluated of bone formation. The follow up period in this study ranges from a minimum of 3 months to maximum of 6 months.

The results are summarized in the tabular column(Table 1)

\begin{tabular}{|l|l|l|l|l|l|l|l|l|}
\hline $\begin{array}{l}\text { Sr } \\
\text { No }\end{array}$ & $\begin{array}{l}\text { Age/ } \\
\text { Sex }\end{array}$ & Etiology & $\begin{array}{l}\text { Pre operative } \\
\text { Body } \\
\text { lengthening } \\
\text { required } \\
\text { (cm) }\end{array}$ & $\begin{array}{l}\text { Pre operative } \\
\text { Ramus } \\
\text { lengthening } \\
\text { required } \\
\text { (cm) }\end{array}$ & $\begin{array}{l}\text { Direction } \\
\text { vector } \\
\text { distraction }\end{array}$ & $\begin{array}{l}\text { Post operative } \\
\text { for } \\
\text { body } \\
\text { lengthening } \\
\text { achieved } \\
\text { (cm) }\end{array}$ & $\begin{array}{l}\text { Post } \\
\text { Operative } \\
\text { ramus } \\
\text { lengthening } \\
\text { achieved } \\
\text { (cm) }\end{array}$ & $\begin{array}{l}\text { Post operative } \\
\text { Facial } \\
\text { esthetics }\end{array}$ \\
\hline 1 & $20 / \mathrm{M}$ & $\begin{array}{l}\text { Unilateral right } \\
\text { TMJ Ankylosis }\end{array}$ & $10 \mathrm{~mm}$ & $15 \mathrm{~mm}$ & Oblique & $12 \mathrm{~mm}$ & $12 \mathrm{~mm}$ & good \\
\hline 2 & $28 / \mathrm{M}$ & $\begin{array}{l}\text { Unilateral left } \\
\text { TMJ Ankylosis }\end{array}$ & $12 \mathrm{~mm}$ & $13 \mathrm{~mm}$ & Oblique & $12 \mathrm{~mm}$ & $13 \mathrm{~mm}$ & satisfactory \\
\hline 3 & $30 / \mathrm{M}$ & $\begin{array}{l}\text { Unilateral right } \\
\text { TMJ Ankylosis }\end{array}$ & $11 \mathrm{~mm}$ & $14 \mathrm{~mm}$ & Oblique & $11 \mathrm{~mm}$ & $14 \mathrm{~mm}$ & good \\
\hline 4 & $25 / \mathrm{F}$ & $\begin{array}{l}\text { Unilateral left } \\
\text { TMJ Ankylosis }\end{array}$ & $10 \mathrm{~mm}$ & $15 \mathrm{~mm}$ & Oblique & $10 \mathrm{~mm}$ & $15 \mathrm{~mm}$ & good \\
\hline
\end{tabular}



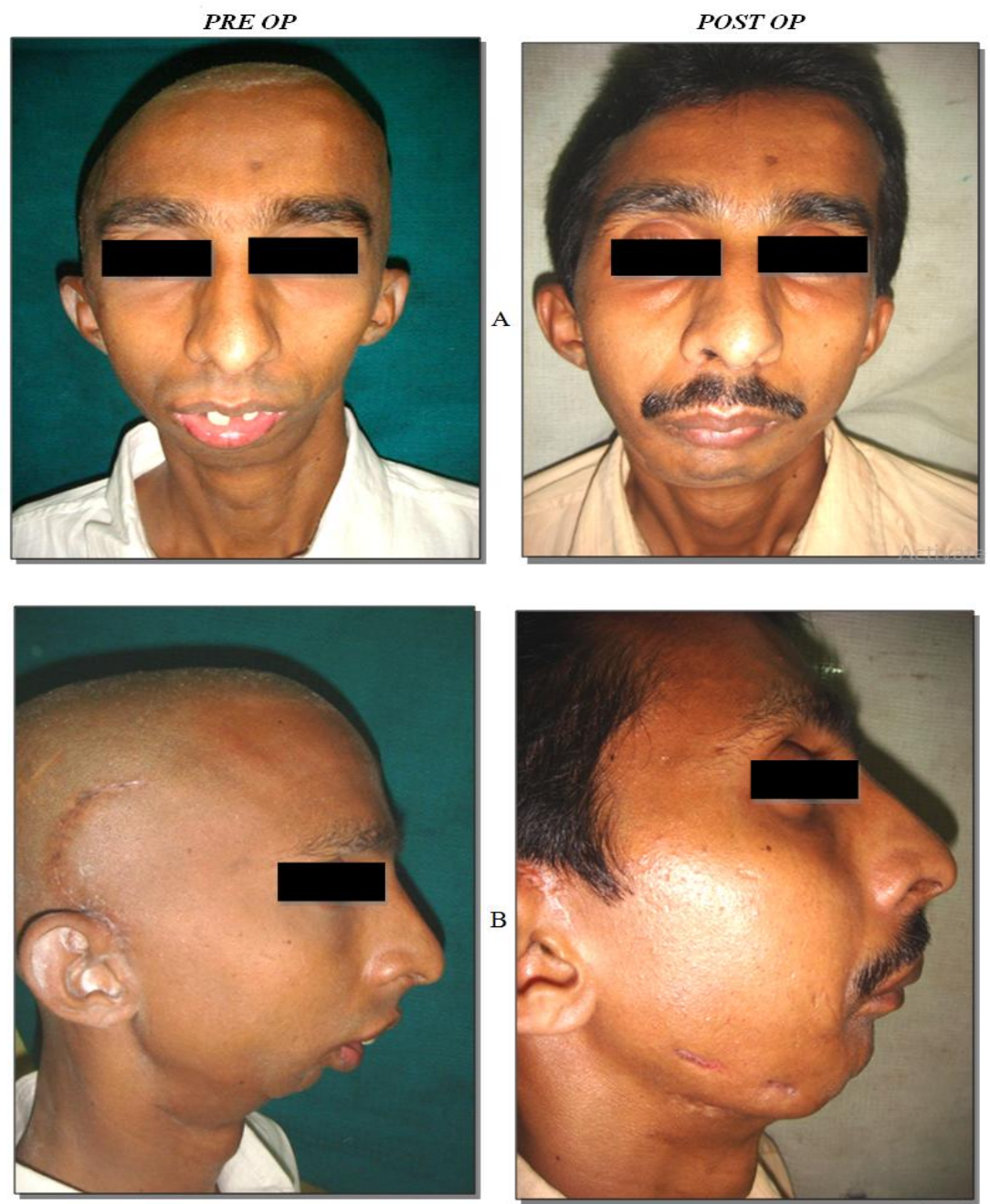

Fig 3, A. Frontal View, B. Lateral View,

\section{Discussion}

Condylar damage during early childhood can produce ankylosis and alteration of the mandibular growth .[8]As a result of destruction of the growth centre and limited mobility of the mandible, gross changes occurs in mandibular shape and size, as well as the surrounding functional matrix. The morbid anatomy in such patients can be as summarized by El sheikh MM [5] et al (1997) : formation of abnormal osseous mass on the affected side of tmj, elongated coronoid process ,reduced posterior facial height, marked ante-gonial notch, crowding of teeth, fullness on the unaffected side, deep bite, chin deviated towards he affected side. The pathogenesis of mandibular hypoplasia caused by TMJ ankylosis is different than that of hemifacialmicrosomia. In hemifacialmicrosomia, there is likely modification of the embryogenesis and intrinsic nature of the tissues, where as in mandibular hypoplasia due to ankylosis, the cause is usually an acquired trauma that induces a series of related chain of deformities. Therefore on restoring the correct form and function of the soft as well as bony tissues, the mandible would regain normal growth potential in ankylosis.[6]

It is known that maxillofacial skeleton development is influenced by the functional matrix so that mandibular growth occurs secondarily in response to demands of the attached neuromuscular envelope and functioning spaces. The bone would grow in response to these stimuli. The deformity in the ankylotic patient is the result of combined effects of the lack of growth of the condylar epiphysis and the absence of stimulus from the functional matrix. The deformity thus will be more severe in those cases with earlier condylar damage and in those cases in which treatment is largely deferred.[10] Mandibular hypoplasia associated with ankylosis were initially treated with the objective of obtaining good joint function with reinstitution of the facial asymmetry was left for a second procedure.[8] Simultaneous reconstruction of the facial asymmetry and the joint surgery for ankylosis were also carried out as a treatment options by various pioneers in these patients. [5,9] This protocol, with some modification, has been used widely for the treatment of mandibular hypoplasia associated with ankylosis. This classical treatment has some disadvantagessuch as donor site morbidity, unpredictable quantity of graft growth, eventual need for intermaxillary fixation and blood transfusions. Bilateral sagittal 
splitting osteotomy of the ramus, camouflage genioplasty, sliding genioplasty, vertical ramus osteotomy and lefort I ostetomy in adults have been described in the literature an ancillary procedures for mandibular deformity correction. In mandibular hypoplasia with ankylosis, a shortening (atrophy) of the adjacent muscle exists. When the treatment of the hypoplasia is accomplished through the placement of graft or mandibular advancement by these above mentioned methods, a rapid expansion of the atrophic facial musculature is produced creating a tension over the advanced segment. This in turn can hamper the desired mandibular lengthening and relapse is easier, as noted in many studies where early relapse is seen with advancement following BSSO.[10]

Correction using orthognathic surgical procedures is applicable on cases of minimal ramus / body deficiency, therefore limited amount of advancement can only be carried out using these techniques. Moreover the abnormally short ramus is rendered shorter after resection of ankylosed joints with the coronoid process, these two factors will leave a residual ramus unfit for saggital splitting.[14] Recently DO has been proposed for the treatment of bony TMJ ankylosis. It is defined as "the regeneration of bone between vascularised bone surfaces that are separated by gradual distraction".[11] It offers several advantages over traditional surgical techniques for mandibular lengthening like, forces generated during distraction of callus are physiologic that leads to proper development of mandible, the amount of bone lengethening can be controlled by proper direction, adaptation of the muscles and surrounding soft tissues is obtained, it avoids the donor site morbidity and relapse, and less invasive.[13]

DO can be carried out by internaland external devices. The device used in the present study was external bone borne distraction device. There are certain advantages of using external devices over the internal devices such as controlled and greater amount of bone lengethening is possible, easy and simple for manipulation, can be removed easily even without giving local anaesthesia and also surgical procedure is avoided in compare to internal device. The only disadvantages are apprehension of external device due to social inconvenience and potential for scars. Mc Carthy and coworkers demonstrated the feasibility of DO for correction of mandibular hypoplasia and did first clinical trial of distraction on human in 1992.Then, various other researchers demonstrated the usefulness, feasibility and indicated distraction as treatment of choice for mandibular hypoplasia, either due to hemifacialmicrosomia or due to other acquired causes like TMJ ankylosis. By many researchers DO was performed simultaneously with gap arthroplasty as a single procedure for TMJ ankylosis and secondary mandibular deformity with successful result.[6,14]

In the recent years, some researchers found that in single stage simultaneous distraction it is difficult to do active physical therapy post operatively due to which possibility of relapse (reankylosis) exists. Moreover it is impossible to exactly predict the mandibular position after condylectomy and almost impossible to control the occlusal plane post arthroplasty which is an important criteria necessary for vector placement'.Therefore in order to overcome these difficulties, they advised staged surgical procedure with DO carried out as a second procedure after achieving stabilized mouth opening and function post interpositional arthroplasty as a first operation in TMJ ankylosis.[15] After reviewing the literature, we concluded following criteria of treatment protocol selected for our patient,First,Staged surgical procedure was used for secondary mandibular deformity correction, with first operation planned to correct mouth opening and attain function using interpositioning arthroplasty with temporalis myofasical flap with active physiotherapy following post operative period. Interpositioning arthroplasty was found to be functionally more advantageous than gap arthroplasty alonewith temporalis myofascial flap considered superior than other interpositional materials Secondly,after achieving adequate mouth opening, function and stable occlusal plane. Second operation planned to correct mandibular body deficiency alone or combination of body and ramus deficiency by using uniplanar indigenously designed extra oral appliances placed either uni or bilaterally.

Biomechanical principles should be followed during distraction if both ramus and corpus are planned for distraction.The oblique orientation of the distraction device may be changed to either more vertical or more horizontal, depending on whether the ramus or mandibular body requires more lengthening respectively. We followed the technique advised by Joseph G McCarthy (1992) and Molina(1995), to create an atraumatic bone discontinuity.[7,16] To optimize the response of osteogenic tissue to distraction, a latency period has been suggested for early callus formation (mesenchymal tissue reaction).Different latency periods has been suggested, ranging from 5-21 days have been reported in clinical studies and animal experiments.A latency period of 7 dayswas given for all the patients is our study. On the eight post operative day we started distraction.[7] In our study we did an advancement of $1 \mathrm{~mm}$ per day according to the principles of illizarov. A consolidation period of 6-8 weeks following the desired advancement by distraction is advised. The device to be removed after radiographic evidence of bone formation. A periodic follow up to assess the changes following distraction is mandatory.In the present study, the device was removed after the radiographic evidence of bone formation was seen that is approximately after 6-8 weeks. All the patients were kept under periodic observation.As concluded by various researchers in their study, we do find expansion of soft tissue over affected side following distraction osteogenesis, with improved facial esthetics[Figure 3]. 
Post ankylotic secondary deformity correction of the mandible always have a challenging situation for the surgeons. DO has proved to be a efficient way of correcting such deformity \& gained significant momentum $\&$ popularity due to its unique advantages like low risk with decreased morbidities, simple manipulation, high curative rate, lower rate of relapse with stable results. The skeletal distraction proceeds parallel to an expansion of all the soft tissues of the maxillofacial region, achieving superior esthetic results to those obtained by other skeletal or soft tissue surgery done alone or in combination. DO is not a one step procedure \& requires careful planning. If the vectors are accurately designed with carefully carried out osteotomies, esthetic and functional results obtained are superior compared to conventional orthognathic procedures. We can plan distraction as a definitive treatment in cases with isolated mandibular hypoplasia. The orthodontist must work together with the surgeon in a well coordinated team to obtain optimal long term results. Patient compliance during the entire treatment period is essential and thus careful patient selection and adequate motivation is utmost important. Although the number of cases and the period of follow up is minimal in our study, this technique of using indigeniously designed extra oral distraction device for correcting mandibular deformities has shown successful results.We conclude by saying that, distraction osteogenesis can be considered as an effective treatment of choice for the surgical correction of unilateral or bilateral hypoplasias of mandible. However to know the long term effects of distraction, periodic follow up is essential.

\section{References}

[1]. Bradley P, James D, Norman J.E.B: Injuries of the condylar \& coronoid processes.J.L.L. Williams (Ed) Rowe and William's Maxillofacial injuries. London. Churchill Livingstone, P.405:1994.

[2]. Kaban LB, Perrot DH, Fisher A: A protocol for management of temporomandibular joint ankylosis. J Oral maxillofacsurg 48, 1145, 1990

[3]. Kwon TG, Park HS, Kim JB et al: Staged surgical treatment for temporomandibular joint ankylosis: Intraoral distraction after temporalis muscle flap reconstruction.J Oral maxillofacSurg 1680-1683, 2006.

[4]. Dean A, Alamillos F: Mandibular distraction in temporo -mandibular joint Ankylosis. PlastReconstrSurg 104, 2021-2031, 1999.

[5]. El-Sheikh MM, Medra AM: Management of unilateral temporomandibular ankylosis associated with facial asymmetry. J Craniomaxillofacsurg Jun;25(3),109-15,1997.

[6]. Dean A, Alamillos F: Mandibular distraction in temporo-mandibular joint Ankylosis. PlastReconstrSurg 104, 2021-2031, 1999.

[7]. McCarthy JG, Schreiber J, Karp N: Lengthening the human mandible by gradual distraction. Plastreconstrsurg 89,1-8, 1992.

[8]. HeggieAA: Concepts in the management of temporo mandibular ankylosis. Ann R. Australascoll Dent Surg 13,132-135, 1996

[9]. Egyedi P, Wittkampf A: Treatment of unilateral ankylosis of the temporomandibular joint when a class II sekeltal relationship exists. J MaxillofacSurg 13(5), 218-23, Oct 1985.

[10]. Frey Dr, Hatch JP, Van SickelJE: Alteration of the mandibular plane during sagittal split advancement: Short-and long-term stability. Oral Surg Oral Med Oral pathol Oral Radio Endol 104(2), 160-9, Aug 2007.

[11]. Joseph G. McCarthy, M.D: Distraction of the craniofacial skeleton Springer - verlay New York Inc. 1999.

[12]. El-Sheikh MM, Medra AM, Warda MH: Bird face deformity secondary to bilateral temporo mandibular joint ankylosis.JCraniomaxillofacSurg 24, 96, 1996.

[13]. Imola MT, Hamlar DD, Thatcher G et al: The versatility of distraction osteogenesis in craniofacial surgery. Arch Facial plastSurg 4(1),8-19, Jan-Mar 2002

[14]. Douglas LR, Douglass JB, Smith P: Intraoral mandibular distraction osteogenesis in a patient with severe micrognathia secondary to TMJ ankylosis using a tooth \& bone-anchored device (PIT device): a case report. J Oral maxillofacSurg 58, 1429-1433, 2000.

[15]. Kwon TG, Park HS, Kim JB et al: Staged surgical treatment for temporomandibular joint ankylosis: Intraoral distraction after temporalis muscle flap reconstruction.J Oral maxillofacSurg 1680-1683, 2006.

[16]. Molina F, Ortiz Monasterio F: Mandibular elongation \& remodelling by distraction: A farewell to major osteotomies. PlastreconstrSurg 96, 825, 1995. 